\title{
The Effect of Exercise Intensity on Total PYY and GLP-1 in Healthy Females: A Pilot Study
}

\author{
Jillian R. Hallworth, ${ }^{1}$ Jennifer L. Copeland, ${ }^{1}$ Jon Doan, ${ }^{1}$ and Tom J. Hazell ${ }^{2}$ \\ ${ }^{1}$ Department of Kinesiology and Physical Education, University of Lethbridge, Lethbridge, AB, Canada T1K $3 M 4$ \\ ${ }^{2}$ Department of Kinesiology and Physical Education, Wilfrid Laurier University, Waterloo, ON, Canada N2L 3C5 \\ Correspondence should be addressed to Tom J. Hazell; thazell@wlu.ca
}

Received 12 July 2016; Revised 4 October 2016; Accepted 19 January 2017; Published 13 February 2017

Academic Editor: Maurizio Muscaritoli

Copyright (c) 2017 Jillian R. Hallworth et al. This is an open access article distributed under the Creative Commons Attribution License, which permits unrestricted use, distribution, and reproduction in any medium, provided the original work is properly cited.

\begin{abstract}
We compared the acute response of anorexigenic signals (total PYY and GLP-1) in response to submaximal and supramaximal exercise. Nine females completed three sessions: (1) moderate-intensity continuous training (MICT; $30 \mathrm{~min} ; 65 \% \mathrm{VO}_{2 \max }$ ); $(2$ ) sprint interval training (SIT; $6 \times 30 \mathrm{sec}$ "all-out" cycling sprints with 4 min recovery); or (3) control (CTRL; no exercise). PYY and GLP-1 were measured via blood samples drawn before, immediately after, and $90 \mathrm{~min}$ after exercise. Perceptions of hunger were rated using a visual analogue scale at all blood sampling time points. There was a session $\times$ time interaction for GLP-1 $(p=0.004)$ where SIT and MICT $(p<0.015$ and $p<0.001)$ were higher compared to CTRL both immediately and 90 min after exercise. There was a main effect of time for PYY where $90 \mathrm{~min}$ after exercise it was decreased versus before and immediately after exercise. There was a session $\times$ time interaction for hunger with lower ratings following SIT versus MICT $(p=0.027)$ and CTRL $(p=0.031)$ 90 min after exercise. These results suggest that though GLP-1 is elevated after exercise in women, it is not affected by exercise intensity though hunger was lower 90 min after exercise with SIT. As the sample size is small further study is needed to confirm these findings.
\end{abstract}

\section{Introduction}

In order to develop evidence-based guidelines for weight management, it is important to understand how exercise affects energy balance (energy intake-energy expenditure). While increasing energy expenditure through exercise seems like a viable strategy to generate an energy deficit, its effectiveness on subsequent weight loss in the absence of dietary restriction is often less than desired $[1,2]$ and though many exercise interventions improve body composition in males, they are less effective in females [2-4]. This may be a result of exercise causing a compensatory increase in energy intake, preventing the energy deficit required for an improvement in body composition [5] as females may partially compensate $(\sim 30 \%)$ for varying levels of exerciseinduced energy expenditure by increasing energy intake [4]. Exercise protocols that can increase energy expenditure without increasing energy intake could be a viable way to develop and improve weight management guidelines.
One such exercise protocol may be sprint interval training (SIT), which is an intense form of high-intensity interval training (HIIT) involving brief $30 \mathrm{sec}$ "all-out" efforts followed by 4 min of active rest $[6,7]$ and has demonstrated similar physiological adaptations to traditional moderateintensity $\left(50-75 \% \mathrm{VO}_{2 \max }\right)$ continuous training (MICT) completed for much longer duration (30-60 min). Despite its vastly reduced training volume [8], SIT has many health benefits [9] including potential reductions in body fat $[8,10]$. While these fat mass losses may be attributed to a protracted increase in postexercise metabolism $[8,11,12]$ or an increase in fat utilization [11,13], others have questioned whether these mechanisms could fully explain the magnitude of fat loss [14]. Therefore, alternative mechanisms need to be explored such as potential exercise-induced effects on appetite-regulating hormones [15, 16] and subsequent energy intake [17].

At present, little is known about the acute effect of exercise on appetite-regulating hormones and the resulting perception of hunger in women. The regulation of energy 
intake is a complex pathway that includes meal initiation, meal termination, meal frequency, and nutrient intake as well as long-term regulation of energy intake in relation to body energy requirement [18]. Each of these components are experienced and acted upon as a result of the integration of various physiological and psychological factors, including hormonal signaling. Important anorexigenic signals, peptide tyrosine-tyrosine (PYY) and glucagon-like peptide-1 (GLP1 ), are mainly involved in the potential satiating effect of exercise $[15,16]$. A change in these hormones stimulates a cascade of events eventually terminating at neurotransmitter receptors in the hypothalamus, affecting appetite and subsequent energy intake. Though several studies have found increases in GLP-1 and PYY with MICT in males [19-22], only a few have examined the responses of these hormones to SIT $[13,23]$. In females, concentrations of PYY and GLP1 have been shown to increase following MICT [24-26], though no effect of exercise intensity was shown at submaximal intensities in highly trained females [25]. However, to date, no study has examined the response of anorexigenic signals to supramaximal SIT in females. While exercise is an effective method of increasing energy expenditure and is often encouraged for weight management, its efficacy in appetite suppression and weight loss may vary with exercise intensity.

Considering that exercise may influence appetiteregulating hormones through several mechanisms, many of which may be intensity-dependent [15], comparing the hormonal response to exercise at different intensities (submaximal and supramaximal) in females is necessary. Thus, the aim of the present study was to compare the total PYY, GLP-1, and hunger response between a traditional moderate-intensity aerobic exercise session and a SIT exercise session in females. We hypothesized that females would have an increased PYY and GLP-1 response to SIT due to the more intense stimulus [15].

\section{Materials and Methods}

2.1. Participants. Thirteen healthy, active females volunteered and provided written informed consent to participate. Four participants withdrew from the study, specifically, two due to lack of time, one who moved cities, and one where there were problems with obtaining two blood draws. Therefore, nine participants were available for analysis. The data presented here are part of a larger research project assessing the role of exercise intensity on appetite-regulating hormones. The male data have been previously published in Appetite [27]. All participants were recreationally active which was classified as participating in moderate-intensity exercise for a minimum of $30 \mathrm{~min}$, at least three times per week. None of the participants were smokers or currently taking prescribed medication (aside from hormonal contraceptives including combination pill, progesterone only pill, and intrauterine device), and all were screened for history of diabetes, eating disorder, drug or alcohol abuse, coronary heart disease, food allergies, and medication use (specifically for those known to affect appetite or hypertension or induce weight loss).
To control for phase of menstrual cycle, all participants, including those on oral contraceptives, completed all sessions during the early follicular phase (days 1-10) of the menstrual cycle, based on self-reported onset of menstruation. All were instructed to perform no physical activity or ingest any caffeine for $48 \mathrm{~h}$ prior to any laboratory visit. The University of Lethbridge Human Subject Research Committee approved all experimental procedures in accordance with the ethical standards of the 1964 Declaration of Helsinki.

2.2. Familiarization Session. Prior to the experimental sessions, each participant attended a familiarization session in which they were screened for exclusion criteria and introduced to the study protocol and equipment. Participants were acclimatized to the exercise equipment as well as the types of effort required during the different exercise protocols. Height and weight were measured using a mechanical beam scale (Health-o-meter Professional, Sunbeam Products, Inc., Illinois, USA). Body mass index (BMI) was calculated as body mass $(\mathrm{kg})$ over height squared $\left(\mathrm{m}^{2}\right)$. Body density was calculated from skinfold measures obtained using a seven-site formula [28] and the Siri equation to determine body fat percentage. Participants then completed a maximal oxygen uptake $\left(\mathrm{VO}_{2 \max }\right)$ with the gas exchange measured directly using an online breath-by-breath gas collection system (Quark CPET, Cosmed, Chicago, Illinois, USA). The test followed a graded protocol to exhaustion on a mechanically braked cycle ergometer (Model 874-E, Monark Exercise, Stockholm, Sweden). Following a $5 \mathrm{~min}$ warm-up at $70 \mathrm{rpm}$ and $1 \mathrm{~kg}$ resistance, participants maintained a $70 \mathrm{rpm}$ cadence with $0.5 \mathrm{~kg}$ resistance added every $2 \mathrm{~min}$ until volitional fatigue was reached or $70 \mathrm{rpm}$ could no longer be maintained. Heart rate $(\mathrm{HR})$ was measured throughout the test using a Polar HR monitor (FT7, Polar Electro Oy, New York, USA) and ratings of perceived exertion (RPE) were assessed simultaneously with each increase of resistance throughout the duration of the test [29]. Verbal encouragement was provided throughout the test. At the end of the test, $\mathrm{VO}_{2 \max }$ (greatest $30 \mathrm{sec}$ average) was established by the presence of a plateau in VO2 values $\left(<1.35 \mathrm{~mL} \cdot \mathrm{kg}^{-1}\right.$ increase in $\left.\mathrm{VO}_{2}\right)$ or when two of the following criteria were obtained: (1) a respiratory exchange ratio (RER) value $>1.15$, (2) HR within $\pm 10 \mathrm{bpm}$ of age predicted maximum HR (220-age), and/or (3) visible subject exhaustion [30]. Upon determination of $\mathrm{VO}_{2 \max }, 65 \%$ of this value was calculated as the target exercise intensity during the MICT session.

2.3. Experimental Sessions. At least one week after the familiarization session, participants completed a randomized three-way crossover study with all experimental sessions (Figure 1) performed at least 1 week apart. The three experimental sessions consisted of 30 minutes of (1) moderateintensity continuous training (MICT; 65\% $\mathrm{VO}_{2 \max }$ ); (2) sprint interval training (SIT); and (3) no exercise control (CTRL). For $24 \mathrm{~h}$ prior to the first experimental session, participants completed a $24 \mathrm{~h}$ dietary journal where they recorded their food intake, including the quantity of each food and beverage consumed for each meal. Participants were 


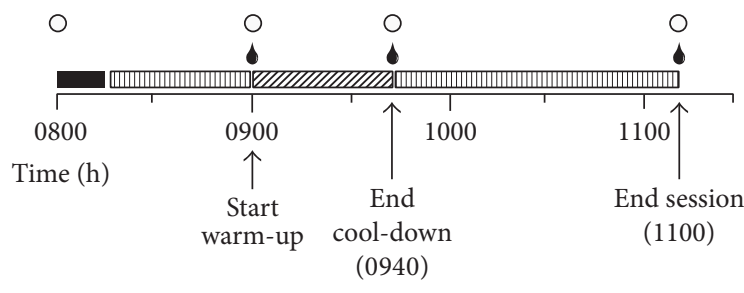

$$
\begin{array}{ll}
\text { VAS } & \text { ② Exercise } \\
\text { Blood sample } & \text { 四 Rest } \\
\text { Breakfast } &
\end{array}
$$

FIGURE 1: Overview of experimental session. VAS: visual analogue scale.

asked to replicate this same diet for the $24 \mathrm{~h}$ prior to each subsequent experimental session. On the morning of each session, participants arrived at the laboratory at $0800 \mathrm{~h}$ in a fasting state (no food or drink except water for a minimum of $10 \mathrm{~h}$ ) and remained in the laboratory for the next $\sim 3 \mathrm{~h}$. Upon arrival for each session participants randomly drew a slip of paper to determine what session they would be doing that day (SIT, MICT, or CTRL). Participants consumed a standardized breakfast $\left(4 \mathrm{kcal} \cdot \mathrm{kg}^{-1} ; 16.7 \mathrm{~kJ} \cdot \mathrm{kg}^{-1}\right)$ consisting of an energy bar (up to $1050 \mathrm{~kJ}$; Clif Bar \& Company, California, USA), plain Quaker rice cake (up to an additional $147 \mathrm{~kJ}$; PepsiCo, Ontario, Canada), and natural peanut butter (up to remaining allotted kJ; Costco Wholesale, Washington, USA). Participants were provided $15 \mathrm{~min}$ to consume breakfast. Water was provided ad libitum throughout experimental sessions. After allowing sufficient time for digestion ( $45 \mathrm{~min})$, the exercise began at $0900 \mathrm{~h}$ and included a $5 \mathrm{~min}$ standardized warm-up, a $30 \mathrm{~min}$ exercise protocol (27 min for SIT with an additional $3 \mathrm{~min}$ rest prior to warm-up to match), and a $5 \mathrm{~min}$ cool-down. Upon completion of exercise $(0940 \mathrm{~h})$, participants remained in the laboratory for an additional $90 \mathrm{~min}$ and rested quietly (reading or using a laptop). Venous blood samples were obtained (detailed below) at $0900 \mathrm{~h}$ (before exercise), $0940 \mathrm{~h}$ (immediately after exercise), and $1110 \mathrm{~h}$ (90 min after exercise). Perceptions of hunger were assessed before breakfast and at each blood sampling timepoint $[31,32]$. Participants were asked to indicate their current level of hunger ("How hungry do you feel?") on a visual analogue scale (VAS) that was anchored at each end with contrasting statements (i.e., "I am not hungry at all" and "I have never been more hungry"). A hedonic scale was not used in the current study as with a crossover design the hedonic levels would be matched across conditions. Identical procedures were followed during the CTRL session with the exception of the exercise period (0900-0940 h) during which participants rested quietly (i.e., reading or using a laptop).

2.4. Exercise Protocols. Both exercise sessions were performed on a cycle ergometer (model 874-E, Monark Exercise, Stockholm, Sweden) and heart rate was recorded continuously using a HR monitor (as described above). The MICT session consisted of $30 \mathrm{~min}$ of continuous cycling at
$65 \% \mathrm{VO}_{2 \max }$ where work rate was determined individually using the American College of Sports Medicine's metabolic equation for gross $\mathrm{VO}_{2}$ during leg cycling, specifically $\mathrm{VO}_{2}=$ (work rate (watts) (body mass $\left.\left.(\mathrm{kg})^{-1}\right)\right) \cdot 10.8+7$. Gas exchange was measured for $1 \mathrm{~min}$ every 5 th minute of exercise using an online breath-by breath gas collection system (described above) and work rate modifications were made accordingly. The SIT protocol was structured as six $30 \mathrm{~s}$ "all-out" efforts against $10 \%$ of body mass, separated by $4 \mathrm{~min}$ recovery periods. Oxygen consumption was not measured during SIT, though we have measured this previously $[33,34]$. Instructions to begin pedaling as fast as possible against the inertial resistance of the ergometer were given prior to each bout and the appropriate load was applied instantaneously (within $3 \mathrm{sec}$ ). Verbal encouragement was provided throughout.

2.5. Blood Processing. All blood samples were collected via venipuncture from the antecubital vein while participants were in a seated position. Venous blood samples were collected into prechilled $6 \mathrm{~mL}$ EDTA tubes (Becton Dickinson Vacutainer K2 EDTA [lavender top] tubes, New York, USA). A protease inhibitor cocktail (BioTool, Ontario, Canada) was added to a final $1 \mathrm{x}$ concentration to prevent hormone degradation. Samples were centrifuged at $1780 \mathrm{rpm}$ for $10 \mathrm{~min}$ at $4^{\circ} \mathrm{C}$ and supernatants were aliquoted, stored at $-80^{\circ} \mathrm{C}$, and batch analyzed at the end of the study to reduce interand intra-assay variability. The concentration of PYY in the plasma samples was determined using Millipore human PYY total ELISA kits (EMD Millipore PYY Total ELISA Kit, Millipore Corporation, Billerica, MA, USA) and the concentration of GLP-1 was determined using Millipore GLP1 total ELISA kits (EMD Millipore GLP-1 Total ELISA Kit, Millipore Corporation, Billerica, MA, USA) following the manufacturer's instructions. The sensitivity of the assays was $1.4 \mathrm{pg} \cdot \mathrm{mL}^{-1}$ for PYY and $1.5 \mathrm{pmol} \cdot 50 \mu \mathrm{L}^{-1}$ for GLP-1. All samples were assayed in duplicate and intra-assay variation was $5.8 \pm 1.9 \%$ and $4.5 \pm 2.3 \%$ for GLP- 1 and PYY, respectively, and interassay variation was $8.0 \pm 4.8 \%$ and $7.3 \pm 3.6 \%$ for GLP1 and PYY, respectively.

2.6. Statistical Analysis. All data were analyzed using the Statistical Package for Social Sciences (SPSS) software v22.0 for Windows (SPSS, Chicago, IL). Hormone concentrations (GLP-1 and PYY) were analyzed as absolute change in concentration from baseline using separate $3 \times 3$ repeated measures ANOVA (session $\times$ time). For significant main effects, a Bonferroni adjustment was used for multiple pairwise comparisons. With regard to the anorexigenic hormones, both absolute concentrations and relative changes from baseline resulted in similar statistical output; therefore subsequent presentation of data will focus on the change in hormone concentrations relative to baseline. For differences in perceptions of hunger, a $3 \times 3$ repeated measures ANOVA (session $\times$ time) was performed using the preexercise measure as baseline. This subjective data was also normalized to baseline values and analyzed as a change from baseline. A one-way repeated measures ANOVA was used to compare AUC values for both hormones. Effect sizes were calculated using Cohen's $d$ to 
TABle 1: Participant characteristics.

\begin{tabular}{lc}
\hline Age $(\mathrm{y})$ & $30.5 \pm 7.9$ \\
Height $(\mathrm{cm})$ & $1.75 \pm 0.15$ \\
Weight $(\mathrm{kg})$ & $72.4 \pm 2.0$ \\
BMI $\left(\mathrm{kg} \cdot \mathrm{m}^{2}\right)$ & $23.5 \pm 2.8$ \\
Body fat $(\%)$ & $22.8 \pm 4.3$ \\
$\mathrm{VO}_{2 \max }\left(\mathrm{mL} \cdot \mathrm{kg}^{-1} \cdot \mathrm{min}^{-1}\right)$ & $40.7 \pm 5.4$ \\
\hline
\end{tabular}

determine the magnitude of an effect independent of sample size. Small effect sizes are considered as $d<0.2$, moderate effect sizes as $d=0.2-0.8$, and large effects sizes as $d>0.8$. Statistical significance was set at $p<0.05$. All results are presented as mean \pm standard deviation.

\section{Results}

3.1. Participants. Nine females completed all experimental sessions and participant characteristics are presented in Table 1.

3.2. Exercise Intensity. During the MICT session, $\mathrm{VO}_{2}$ reached steady state at $\sim 65 \%$ of $\mathrm{VO}_{2 \max }(27.37 \pm$ $\left.3.0 \mathrm{~mL} \cdot \mathrm{kg}^{-1} \cdot \mathrm{min}^{-1}\right)$. The peak HR achieved was significantly higher during SIT (171.6 $\pm 8.1 \mathrm{bpm})$ versus MICT $(153.1 \pm 15.2 \mathrm{bpm}, p=0.003)$; however, average HR did not differ significantly between exercise sessions (MICT = $137.3 \pm 14.8 \mathrm{bpm}$; SIT $=135.5 \pm 13.9 \mathrm{bpm}, p=0.774)$.

3.3. Plasma GLP-1 Response to Exercise. Circulating concentrations of total GLP-1 during all three experimental sessions, presented as change from baseline, can be seen in Figure 2. There was a significant session by time interaction $(p=$ $0.004)$ where MICT and SIT were increased versus CTRL (large effect sizes) at immediately $(p<0.001, d=-1.4$; $p=0.002, d=-1.9)$ and $90 \mathrm{~min}$ after exercise $(p<0.001$, $d=-1.2 ; p=0.015, d=-1.0)$, respectively.

3.4. Plasma PYY Response to Exercise. Circulating concentrations of total PYY during all three experimental sessions, presented as change from baseline, can be seen in Figure 3. There was a main effect of time for PYY $(p=0.007)$, where $90 \mathrm{~min}$ after exercise concentrations were lower (moderate to large effect sizes) compared to before exercise $(p=0.016$, $d=-0.9)$ and immediately after exercise $(p=0.013, d=$ $-0.3)$. There was no main effect for session $(p=0.249)$ and no session $\times$ time interaction $(p=0.256)$.

3.5. Hunger Ratings. Perceptions of hunger during all three experimental sessions can be seen in Figure 4. There was a significant session $\times$ time interaction $(p=0.025)$ where SIT was decreased (moderate effect size) versus CTRL and MICT 90 min after exercise $(p=0.027, d=0.06$ and $p=0.031$, $d=0.5$, resp.). There were no other significant differences.

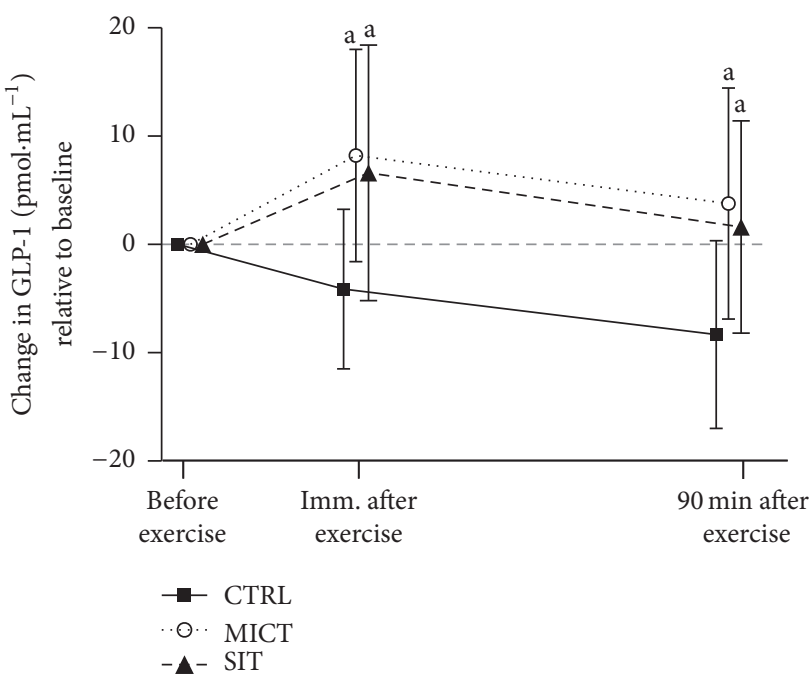

FIGURE 2: Changes in GLP-1 concentrations across all time points relative to baseline for each experimental session. CTRL: control (no exercise); MICT: moderate-intensity continuous training; SIT: sprint interval training. Note. ${ }^{\text {a }}$ Significantly different versus CTRL $(p<0.015)$.

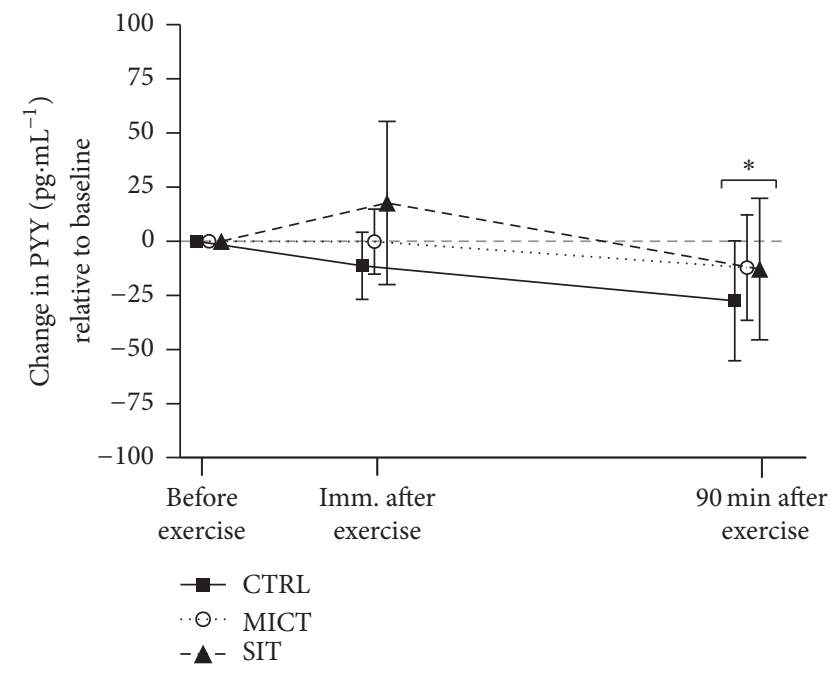

FIgURE 3: Changes in PYY concentrations across all time points relative to baseline for each experimental session. CTRL: control (no exercise); MICT: moderate-intensity continuous training; SIT: sprint interval training. Note. ${ }^{*}$ Significantly different versus before exercise $(p=0.016)$ and immediately after exercise $(p=0.013)$.

\section{Discussion}

To our knowledge, this is the first study to compare the total GLP-1 and PYY response to MICT and SIT among recreationally active females. Our results demonstrate that total GLP-1 increased in response to exercise compared to CTRL with no difference between MICT and SIT. Total PYY concentrations were lower $90 \mathrm{~min}$ after exercise compared to before and immediately after exercise with no difference between sessions. Feeding did not appear to influence the perception of hunger (perhaps the meal was too small), 


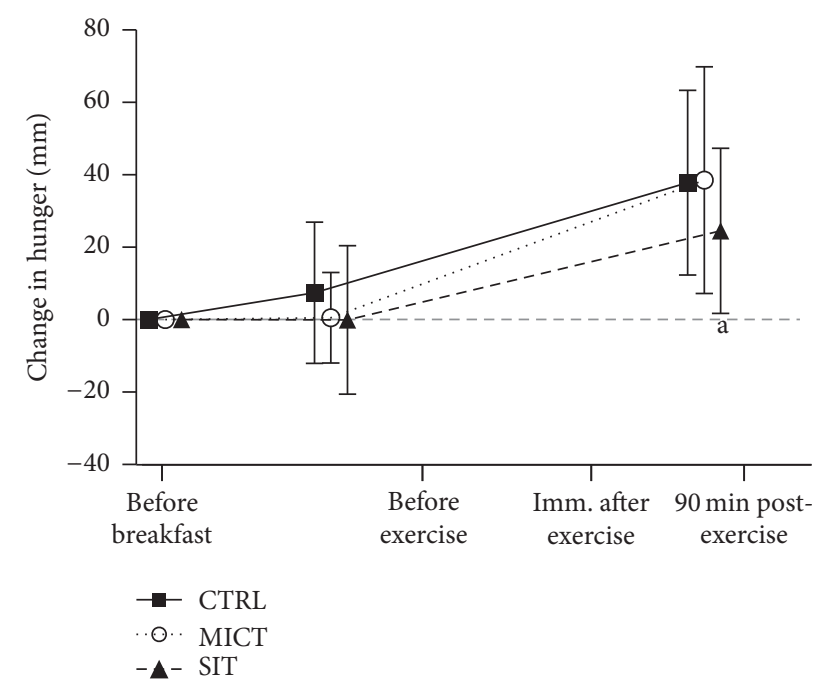

FIgURE 4: Perceptions of hunger at all time points during each experimental session. CTRL: control (no exercise); MICT: moderateintensity continuous training; SIT: sprint interval training. Note. ${ }^{a}$ Significantly different versus CTRL and MICT $(p<0.027)$.

though both MICT and SIT resulted in lower ratings of hunger compared to CTRL after exercise where only SIT still had depressed perceptions of hunger $90 \mathrm{~min}$ after exercise.

The research on the effects of exercise on GLP-1 is not as prominent as other hormones such as PYY, leptin, and ghrelin. Previous studies generally demonstrate that MICT results in an increase in GLP-1 in both males and females [22, $26,35]$. However, several studies have demonstrated no effect of exercise $[13,25,36]$ with no effect of increasing intensity during submaximal continuous exercise [25] and no effect of SIT [13]. Our current results demonstrate that postprandial exercise significantly increased total GLP-1 concentrations after exercise (immediately and $90 \mathrm{~min}$ ) with no difference between MICT and SIT. To our knowledge, this is the first study in females to examine the GLP-1 response to both MICT and SIT and report a significant change in GLP-1 following SIT.

Previous studies have generally shown that MICT $[13,22$, 23, 35-37] and SIT [13, 23, 36] increase PYY after exercise in males. There have been limited studies in females as only two have been completed to date demonstrating increase similar to males following MICT $[24,26]$, though to the authors' knowledge no study has measured the PYY response to SIT in females. We found no effect of exercise (MICT or SIT) on PYY in females as the present results illustrate no effect of session on total PYY, only an effect of time where PYY was lower at $90 \mathrm{~min}$ after exercise compared to before exercise. While our findings are not in agreement with the majority of previous literature there are several other reports of no effect of MICT [21, 38-40] or HIIT [37] on PYY and could involve our exercise being performed in the postprandial state while several other studies are in the fasting state $[19,21,24,36,38,41]$. Some individuals perform exercise after consuming an energy drink or bar and so we believe it is important to examine exercise after eating as well as in a fasting state. It is also possible that differences between studies are explained by differing intensity or duration of exercise, depending on the mechanism by which exercise may influence PYY concentrations.

With no clear understanding of the mechanisms involved in the effect of exercise on appetite-regulation we have recently highlighted several possibilities [15]. Potential increases in sympathetic nervous system activity can influence both GLP-1 and PYY as catecholamines can stimulate the release of both hormones from intestinal L-cells $[42,43]$. Another possibility involves the contractioninduced cytokine interleukin-6 (IL-6), with interesting evidence of its role in increasing GLP-1 in animals [44, 45], though no research has been conducted to date in humans. Elevated IL-6 concentrations have been shown to positively correlate with energy intake in humans [46], though whether this is mediated through GLP-1 remains to be determined. Future work should begin to focus on the potential mechanisms by which exercise alters appetiteregulation in an effort to unravel the complex findings in the literature.

Elevated concentrations of these hormones are often accompanied by decreased perceptions of hunger $[19,22$, 47]. Here we found that both MICT and SIT decrease the perception of hunger immediately after exercise in females, consistent with the increases in GLP-1. However, the perception of hunger dissociates from GLP-1 as the lower hunger ratings were maintained at 90 min after exercise only after SIT, while GLP-1 was still similar between MICT and SIT. There are several possible explanations for this apparent dissociation; the first and simplest is that these hormones are only one factor that can influence appetite and other hormones should be considered such as ghrelin. Second, measuring the active forms of these hormones may have provided an answer more in line with the perception of hunger response. Third, future research could be warranted to see if SIT increases leptin as SIT is known to increase postexercise fat oxidation $[11,13]$. Fourth, perhaps we should have measured the circulating neuropeptides involved in appetite-regulation such as agouti-related peptide (AgRP) and alpha-melanocyte-stimulating hormone [48].

This study provides insight, specifically in females, into appetite-regulating peptides, perceptions of hunger, and how they may vary between exercise intensities that are often prescribed for weight loss. The study does have several limitations. First the sample size is small and included only young, healthy individuals of reproductive age. Further study is needed to confirm these findings and to explore these effects among inactive, obese, or postmenopausal women. Also, we measured total concentrations of both GLP-1 and PYY rather than their bioactive form which may have limited our conclusions [49] though many studies suggest that total concentrations reflect changes in other forms $[13,22,23$, $26,36,41,50,51]$. Furthermore, measuring acylated ghrelin concentrations may have provided additional insight, as this hormone may be more responsive to exercise intensity (Hazell et al., 2016; Schubert et al., 2014). Finally, while participants were instructed to consume the same foods in the $24 \mathrm{~h}$ prior to sessions and to refrain from strenuous exercise 
and alcohol in the hours leading up to the session, their compliance is not guaranteed.

\section{Conclusion}

This is the first study to compare the response of total GLP1, PYY, and perceptions of hunger between MICT and SIT in females. The primary finding of this study is that GLP1 increased after both MICT and SIT though there were no effects of exercise on PYY. Though the similar increases in GLP-1 are in line with the decreased hunger immediately after exercise after both MICT and SIT, only SIT was associated with prolonged suppression of hunger $90 \mathrm{~min}$ after exercise. Future studies should examine the potential mechanisms involved in the exercise-induced changes in GLP-1 and hunger. Additionally, future studies are needed to determine if the acute response of these hormones following exercise translates into altered EI, with potential implication to weight management strategies.

\section{Competing Interests}

The authors declare that they have no competing interests.

\section{Acknowledgments}

The authors would like to thank all participants for taking part in this study and Matthew Schmale for his assistance with data collection. Funding was provided to Tom J. Hazell through a University of Lethbridge Research Fund grant. This work was partially supported by a Queen Elizabeth II Graduate Scholarship to JRH.

\section{References}

[1] J. M. Jakicic, K. Clark, E. Coleman et al., "Appropriate intervention strategies for weight loss and prevention of weight regain for adults," Medicine and Science in Sports and Exercise, vol. 33, no. 12, pp. 2145-2156, 2001.

[2] J. E. Donnelly, J. O. Hill, D. J. Jacobsen et al., "Effects of a 16month randomized controlled exercise trial on body weight and composition in young, overweight men and women: the midwest exercise trial," Archives of Internal Medicine, vol. 163, no. 11, pp. 1343-1350, 2003.

[3] J. P. Després, C. Bouchard, R. Savard, A. Tremblay, M. Marcotte, and G. Thériault, "Effects of exercise-training and detraining on fat cell lipolysis in men and women," European Journal of Applied Physiology and Occupational Physiology, vol. 53, no. 1, pp. 25-30, 1984.

[4] K. R. Westerterp, G. A. L. Meijer, E. M. E. Janssen, W. H. M. Saris, and F. T. Hoor, "Long-term effect of physical activity on energy balance and body composition," British Journal of Nutrition, vol. 68, no. 1, pp. 21-30, 1992.

[5] N. A. King, K. Horner, A. P. Hills et al., "Exercise appetite and $\mathrm{w}$ eight $\mathrm{m}$ anagem ent: understanding the compensatory responses in eating behaviour and how they contribute to variability in exercise-induced weight loss," British Journal of Sports Medicine, vol. 46, no. 5, pp. 315-322, 2012.

[6] M. J. Gibala, J. P. Little, M. van Essen et al., "Short-term sprint interval versus traditional endurance training: similar initial adaptations in human skeletal muscle and exercise performance," Journal of Physiology, vol. 575, no. 3, pp. 901-911, 2006.

[7] T. J. Hazell, R. E. K. MacPherson, B. M. R. Gravelle, and P. W. R. Lemon, "10 or 30-s sprint interval training bouts enhance both aerobic and anaerobic performance," European Journal of Applied Physiology, vol. 110, no. 1, pp. 153-160, 2010.

[8] R. E. K. MacPherson, T. J. Hazell, T. D. Olver, D. H. Paterson, and P. W. R. Lemon, "Run sprint interval training improves aerobic performance but not maximal cardiac output," Medicine and Science in Sports and Exercise, vol. 43, no. 1, pp. 115-122, 2011.

[9] M. J. Gibala, J. B. Gillen, and M. E. Percival, "Physiological and health-related adaptations to low-volume interval training: influences of nutrition and sex," Sports Medicine, vol. 44, Supplement 2, pp. 127-137, 2014.

[10] T. J. Hazell, C. D. Hamilton, T. D. Olver, and P. W. R. Lemon, "Running sprint interval training induces fat loss in women," Applied Physiology, Nutrition and Metabolism, vol. 39, no. 8, pp. 944-950, 2014.

[11] H. H. Chan and S. F. Burns, "Oxygen consumption, substrate oxidation, and blood pressure following sprint interval exercise," Applied Physiology, Nutrition and Metabolism, vol. 38, no. 2, pp. 182-187, 2013.

[12] T. J. Hazell, T. D. Olver, C. D. Hamilton, and P. W. R. Lemon, "Two minutes of sprint-interval exercise elicits 24-hr oxygen consumption similar to that of 30 min of continuous endurance exercise," International Journal of Sport Nutrition and Exercise Metabolism, vol. 22, no. 4, pp. 276-283, 2012.

[13] K. Beaulieu, T. D. Olver, K. C. Abbott, and P. W. R. Lemon, "Energy intake over 2 days is unaffected by acute sprint interval exercise despite increased appetite and energy expenditure," Applied Physiology, Nutrition and Metabolism, vol. 40, no. 1, pp. 79-86, 2014.

[14] C. B. Williams, J. G. E. Zelt, L. N. Castellani et al., "Changes in mechanisms proposed to mediate fat loss following an acute bout of high-intensity interval and endurance exercise," Applied Physiology, Nutrition and Metabolism, vol. 38, no. 12, pp. 12361244, 2013.

[15] T. J. Hazell, H. Islam, L. K. Townsend, M. S. Schmale, and J. L. Copeland, "Effects of exercise intensity on plasma concentrations of appetite-regulating hormones: potential mechanisms," Appetite, vol. 98, pp. 80-88, 2016.

[16] M. M. Schubert, S. Sabapathy, M. Leveritt, and B. Desbrow, "Acute exercise and hormones related to appetite regulation: a meta-analysis," Sports Medicine, vol. 44, no. 3, pp. 387-403, 2014.

[17] M. M. Schubert, B. Desbrow, S. Sabapathy, and M. Leveritt, "Acute exercise and subsequent energy intake. A meta-analysis," Appetite, vol. 63, pp. 92-104, 2013.

[18] E. Valassi, M. Scacchi, and F. Cavagnini, "Neuroendocrine control of food intake," Nutrition, Metabolism and Cardiovascular Diseases, vol. 18, no. 2, pp. 158-168, 2008.

[19] D. R. Broom, D. J. Stensel, N. C. Bishop, S. F. Burns, and M. Miyashita, "Exercise-induced suppression of acylated ghrelin in humans," Journal of Applied Physiology, vol. 102, no. 6, pp. 21652171, 2007.

[20] J. A. Cooper, A. C. Watras, C. M. Paton, F. H. Wegner, A. K. Adams, and D. A. Schoeller, "Impact of exercise and dietary fatty acid composition from a high-fat diet on markers of hunger and satiety," Appetite, vol. 56, no. 1, pp. 171-178, 2011.

[21] J. A. King, L. K. Wasse, J. Ewens et al., "Differential acylated ghrelin, peptide YY3-36, appetite, and food intake responses 
to equivalent energy deficits created by exercise and food restriction," Journal of Clinical Endocrinology and Metabolism, vol. 96, no. 4, pp. 1114-1121, 2011.

[22] S.-Y. Ueda, T. Yoshikawa, Y. Katsura, T. Usui, H. Nakao, and S. Fujimoto, "Changes in gut hormone levels and negative energy balance during aerobic exercise in obese young males," Journal of Endocrinology, vol. 201, no. 1, pp. 151-159, 2009.

[23] K. Deighton, R. Barry, C. E. Connon, and D. J. Stensel, "Appetite, gut hormone and energy intake responses to low volume sprint interval and traditional endurance exercise," European Journal of Applied Physiology, vol. 113, no. 5, pp. 1147-1156, 2013.

[24] T. A. Hagobian, M. Yamashiro, J. Hinkel-Lipsker, K. Streder, N. Evero, and T. Hackney, "Effects of acute exercise on appetite hormones and ad libitum energy intake in men and women," Applied Physiology, Nutrition and Metabolism, vol. 38, no. 1, pp. 66-72, 2013.

[25] S. M. Howe, T. M. Hand, D. E. Larson-Meyer, K. J. Austin, B. M. Alexander, and M. M. Manore, "No effect of exercise intensity on appetite in highly-trained endurance women," Nutrients, vol. 8, no. 4, article no. 44, 2016.

[26] D. E. Larson-Meyer, S. Palm, A. Bansal, K. J. Austin, A. M. Hart, and B. M. Alexander, "Influence of running and walking on hormonal regulators of appetite in women," Journal of Obesity, vol. 2012, Article ID 730409, 15 pages, 2012.

[27] T. J. Hazell, H. Islam, J. R. Hallworth, and J. L. Copeland, "Total PYY and GLP-1 responses to submaximal continuous and supramaximal sprint interval cycling in men," Appetite, vol. 108, pp. 238-244, 2017.

[28] A. S. Jackson, M. L. Pollock, and A. Ward, "Generalized equations for predicting body density of women," Medicine and Science in Sports and Exercise, vol. 12, no. 3, pp. 175-182, 1980.

[29] G. A. V. Borg, "Psychophysical bases of perceived exertion," Medicine and Science in Sports and Exercise, vol. 14, no. 5, pp. 377-381, 1982.

[30] A. W. Midgley, L. R. McNaughton, R. Polman, and D. Marchant, "Criteria for determination of maximal oxygen uptake: a brief critique and recommendations for future research," Sports Medicine, vol. 37, no. 12, pp. 1019-1028, 2007.

[31] A. Flint, A. Raben, J. E. Blundell, and A. Astrup, "Reproducibility, power and validity of visual analogue scales in assessment of appetite sensations in single test meal studies," International Journal of Obesity, vol. 24, no. 1, pp. 38-48, 2000.

[32] B. A. Parker, K. Sturm, C. G. MacIntosh, C. Feinle, M. Horowitz, and I. M. Chapman, "Relation between food intake and visual analogue scale ratings of appetite and other sensation in healthy older and young subjects," European Journal of Clinical Nutrition, vol. 58, no. 2, pp. 212-218, 2004.

[33] T. J. Hazell, T. D. Olver, R. E. Macpherson, C. D. Hamilton, and P. W. Lemon, "Sprint interval exercise elicits near maximal peak VO2 during repeated bouts with a rapid recovery within 2 minutes," The Journal of Sports Medicine and Physical Fitness, vol. 54, no. 6, pp. 750-756, 2014.

[34] L. K. Townsend, K. M. Couture, and T. J. Hazell, "Mode of exercise and sex are not important for oxygen consumption during and in recovery from sprint interval training," Applied Physiology, Nutrition and Metabolism, vol. 39, no. 12, pp. 13881394, 2014.

[35] S.-Y. Ueda, T. Yoshikawa, Y. Katsura, T. Usui, and S. Fujimoto, "Comparable effects of moderate intensity exercise on changes in anorectic gut hormone levels and energy intake to high intensity exercise," Journal of Endocrinology, vol. 203, no. 3, pp. 357-364, 2009.
[36] H. Kawano, M. Mineta, M. Asaka et al., "Effects of different modes of exercise on appetite and appetite-regulating hormones," Appetite, vol. 66, pp. 26-33, 2013.

[37] K. Deighton, E. Karra, R. L. Batterham, and D. J. Stensel, "Appetite, energy intake, and PYY3-36 responses to energymatched continuous exercise and submaximal high-intensity exercise," Applied Physiology, Nutrition and Metabolism, vol. 38, no. 9, pp. 947-952, 2013.

[38] L. Balaguera-Cortes, K. E. Wallman, T. J. Fairchild, and K. J. Guelfi, "Energy intake and appetite-related hormones following acute aerobic and resistance exercise," Applied Physiology, Nutrition and Metabolism, vol. 36, no. 6, pp. 958-966, 2011.

[39] M. H.-Y. Cheng, D. Bushnell, D. T. Cannon, and M. Kern, "Appetite regulation via exercise prior or subsequent to high-fat meal consumption," Appetite, vol. 52, no. 1, pp. 193-198, 2009.

[40] M. E. Holmstrup, T. J. Fairchild, S. Keslacy, R. S. Weinstock, and J. A. Kanaley, "Satiety, but not total PYY, Is increased with continuous and intermittent exercise," Obesity, vol. 21, no. 10, pp. 2014-2020, 2013.

[41] D. R. Broom, R. L. Batterham, J. A. King, and D. J. Stensel, "Influence of resistance and aerobic exercise on hunger, circulating levels of acylated ghrelin, and peptide YY in healthy males," American Journal of Physiology-Regulatory Integrative and Comparative Physiology, vol. 296, no. 1, pp. R29-R35, 2009.

[42] T. C. M. Adam and M. S. Westerterp-Plantenga, "Activityinduced GLP-1 release in lean and obese subjects," Physiology and Behavior, vol. 83, no. 3, pp. 459-466, 2004.

[43] S. Brechet, P. Plaisancié, V. Dumoulin, J. A. Chayvialle, J. C. Cuber, and J. Claustre, "Involvement of beta1- and beta2- but not beta3-adrenoceptor activation in adrenergic PYY secretion from the isolated colon," Journal of Endocrinology, vol. 168, no. 1, pp. 177-183, 2001.

[44] H. Ellingsgaard, I. Hauselmann, B. Schuler et al., "Interleukin-6 enhances insulin secretion by increasing glucagon-like peptide1 secretion from L cells and alpha cells," Nature Medicine, vol. 17, no. 11, pp. 1481-1489, 2011.

[45] R. Shirazi, V. Palsdottir, J. Collander et al., "Glucagon-like peptide 1 receptor induced suppression of food intake, and body weight is mediated by central IL-1 and IL-6," Proceedings of the National Academy of Sciences of the United States of America, vol. 110, no. 40, pp. 16199-16204, 2013.

[46] C. Almada, L. R. Cataldo, S. V. Smalley et al., "Plasma levels of interleukin- 6 and interleukin-18 after an acute physical exercise: relation with post-exercise energy intake in twins," Journal of Physiology and Biochemistry, vol. 69, no. 1, pp. 85-95, 2013.

[47] N. A. King, P. Caudwell, M. E. Hopkins et al., "Metabolic and behavioral compensatory responses to exercise interventions: barriers to weight loss," Obesity, vol. 15, no. 6, pp. 1373-1383, 2007.

[48] J. A. Parker and S. R. Bloom, "Hypothalamic neuropeptides and the regulation of appetite," Neuropharmacology, vol. 63, no. 1, pp. 18-30, 2012.

[49] S. S. Hussain and S. R. Bloom, "The regulation of food intake by the gut-brain axis: implications for obesity," International Journal of Obesity, vol. 37, no. 5, pp. 625-633, 2013.

[50] C. Martins, L. M. Morgan, S. R. Bloom, and M. D. Robertson, "Effects of exercise on gut peptides, energy intake and appetite," Journal of Endocrinology, vol. 193, no. 2, pp. 251-258, 2007.

[51] C. Martins, D. Stensvold, G. Finlayson et al., "Effect of moderate- and high-intensity acute exercise on appetite in obese individuals," Medicine and Science in Sports and Exercise, vol. 47, no. 1, pp. 40-48, 2015. 


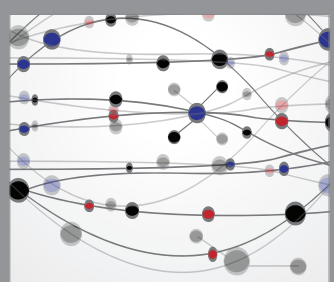

The Scientific World Journal
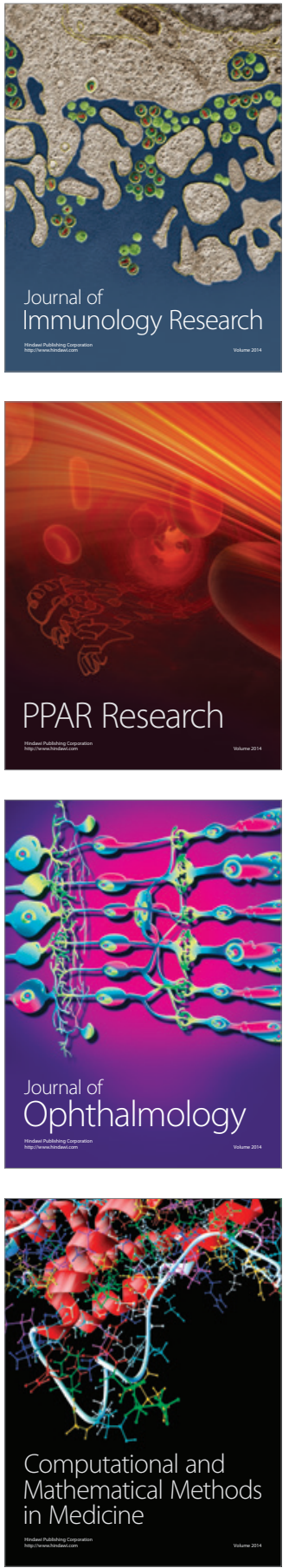

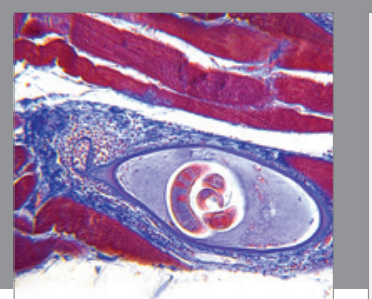

Gastroenterology Research and Practice
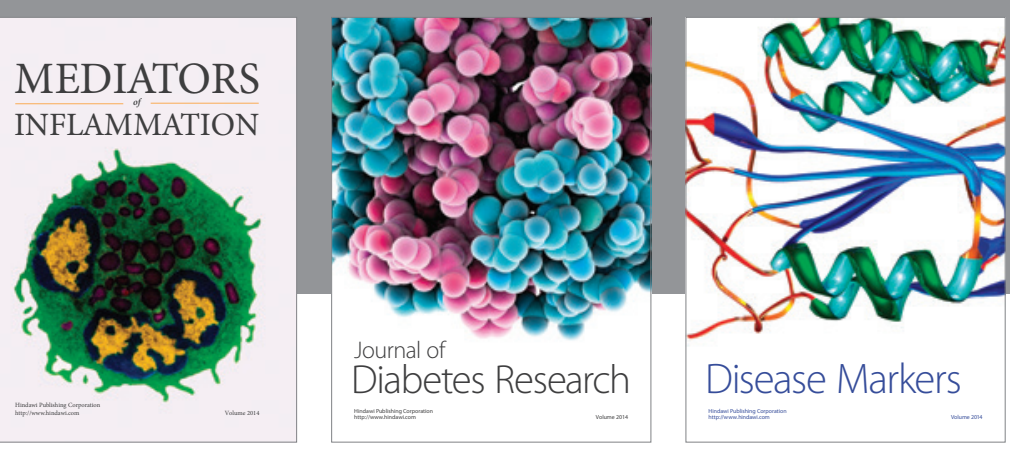

Disease Markers

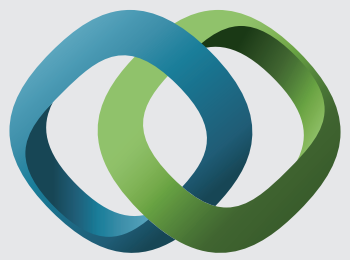

\section{Hindawi}

Submit your manuscripts at

https://www.hindawi.com
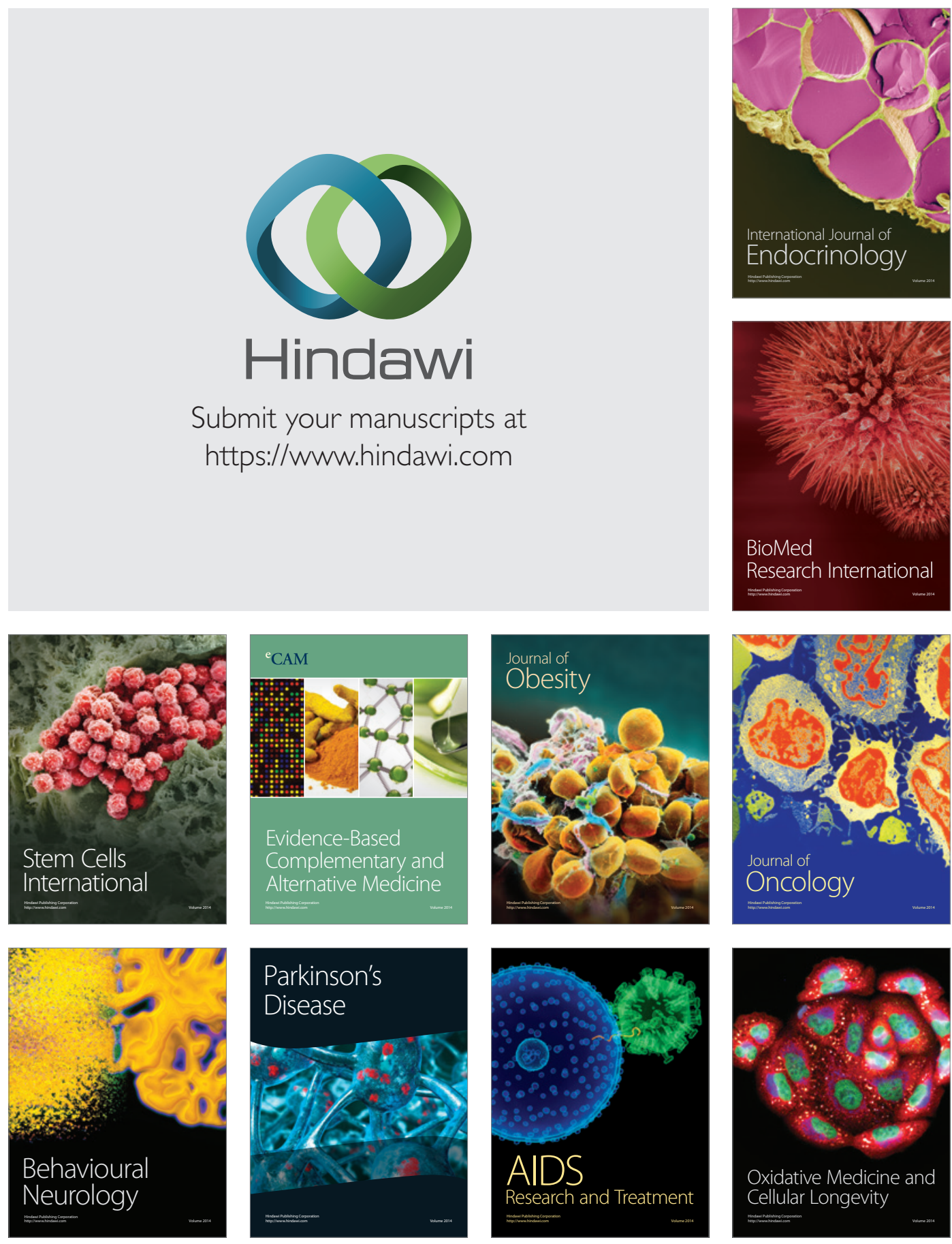